\title{
VOM HOSPITAL ZUM KRANKENHAUS. ZUM UMGANG MIT PSYCHISCH UND SOMATISCH KRANKEN IM FRÜHNEUZEITLICHEN EUROPA
}

Böhlau Verlag, Köln-Weimar 2005

Die vorliegende Arbeit von Carlos Watzka ist aus einer 2004 abgeschlossenen Diplomarbeit hervorgegangen. Im ersten Abschnitt wird die Entwicklung des europäischen Hospital- und Krankenhauswesens in der frühen Neuzeit ausführlich dargestellt. Danach wird die Situation in der Steiermark anhand der vorliegenden Dokumente detailliert beschrieben, wobei auf die Krankenhäuser der Barmherzigen Brüder besonders eingegangen wird. Im Wesentlichen wird dabei die Zeitspanne zwischen 1500 und 1750 behandelt.

Im frühneuzeitlichen Europa hatte sich, wie der Autor schreibt, ein klares Konzept von „Irrsinn“ als Störung des Körpers oder einer Funktion etabliert, die deutlich von Besessenheit unterschieden wurde. Die Terminologie war differenziert, allerdings fehlten für viele Begriffe allgemein anerkannte Definitionen. Watzka entlarvt die negativen Vorstellungen über den „vorpsychiatrischen“ Umgang mit Irren als falsch. Negative Extrembeispiele wurden immer wieder zitiert, wobei die Schaffung von spezialisierten Großkrankenanstalten im 19. Jahrhundert zu keiner wesentlichen Verbesserung für die Kranken führte: die hohen Sterblichkeitsraten in diesen Anstalten weisen auf schlechte Ernährungs- und Lebensbedingungen hin.

Als Auslöser für psychische Krankheit sah man in der frühen Neuzeit somatische Dysfunktionen, ungesunde Lebensweise, starke Affekte, astronomisch-astrologische und mechanisch-chemische Faktoren sowie göttliches und dämonisches Eingreifen. Schon seit der Antike existierten differenzierte Therapieempfehlungen. Die Standardmaßnahmen in der Therapie psychisch Kranker waren Maßnahmen wie Aderlass, starke Abführ- und Brechmittel sowie diätetische Maßnahmen wie die Regelung der Ernährung und der Arbeits-, Wach- und Schlafphasen. Im 18. Jahrhundert wurden zunehmend radikalere Therapievorschläge formuliert, auch die Zahl der Internierungen nahm zu, wobei besonders die „echten Wahnsinnigen“ und Tobenden betroffen waren.

Watzka unterscheidet mehrere Arten von Anstalten, in denen sozial depravierte Personen untergebracht wurden, wobei das Hospital als multifunktionale Unterbringungseinrichtung für als hilfsbedürftig 
wahrgenommene Personen die verbreitetste Form war. Die Betreuung war hier nicht therapeutisch ausgerichtet, ärztliche Leistungen wurden meist nur „zugekauft“. Oft am Rand großer Städte gelegen waren die Hospitäler gut erreichbar und im Seuchenfall doch leicht zu isolieren. Große Häuser erinnerten in ihrer Hallen- oder Basilikaform oft an Sakralbauten, die Krankensäle waren teilweise mit einem Altar ausgestattet. Die meisten Kranken waren in großen Gemeinschaftssälen untergebracht, aus sittlichen Gründen nach Geschlechtern getrennt. Gegebenenfalls dienten kleinere Räume zur Isolation. Die Aufnahme in ein Hospital wurde meist als Glück betrachtet, waren die Bewohner doch in einer Gesellschaft mit knappen ökonomischen Ressourcen vergleichsweise gut versorgt. Psychisch kranke Personen wurden meist nur isoliert, wenn sie als gefährlich oder anstößig betrachtet wurden, sonst waren sie mit den anderen PatientInnen zusammen.

Im zweiten Teil beschreibt Watzka die Entwicklung des therapeutisch orientierten Spitalskonzeptes der barmherzigen Brüder. 1540 von Juan Ciudad in Granada gegründet, breitete sich die von der katholischen Kirche unterstützte Bewegung schnell aus. Um 1700 gab es bereits an die 200 Spitäler der barmherzigen Brüder in Europa, wobei die einzelnen Anstalten meist über 20 bis 40 Betten verfügten, die Aufenthaltsdauer betrug im Durchschnitt wenige Wochen. Die hohe Zahl der jährlich betreuten Patienten führte zu einer hohen gesellschaftlichen Bedeutung der Hospitäler der Barmherzigen Brüder. Sie verfügten über Strukturen, die moderne Krankenhäuser auszeichnen: tägliche gemeinsame Visiten, an denen Ärzte, Chirurgen, Pfleger und Apotheker teilnahmen. Das Führen schriftlicher Aufzeichnungen war genauso vorgeschrieben wie die Beichte und Waschung bei der Aufnahme und die Spitalskleidung der Patienten. Das Ziel war eindeutig die Heilung und Entlassung der Patienten. Der therapeutische Ansatz war breit: Medizin, Ernährung, Gespräche, religiöse Übungen, aber auch Hygienemaßnahmen.

Betreut wurden nur kranke Männer, keine anderen Gruppen von Bedürftigen, sonst gab es keine nationalen oder religiösen Einschränkungen. Die Kranken waren in einem großen Saal mit einem Altar untergebracht. Einzelne kleine Räume dienten unter anderem der Isolierung psychisch Kranker, von denen allerdings die meisten bei den anderen Patienten untergebracht waren. Psychische Krankheit wurde als behandelbar betrachtet. Der Autor kann anhand der Aufzeichnungen belegen, dass drei von vier „Irren“ nach maximal vier Wochen geheilt oder gebessert entlassen werden konnten. Auch die Sterberate der psychisch Kranken war mit 4,9\% nur etwa halb so hoch wie bei somatisch Kranken, was auf gute Ernährung und Betreuung der Patienten schließen lässt. 
Watzka gelingt es, alte Vorurteile über den Umgang mit „Irren“ in der Frühen Neuzeit fundiert zu widerlegen. Das Buch ist dabei äußerst informativ und doch kurzweilig geschrieben. Der Band wird durch einen übersichtlichen Anhang mit Bild- und Kartenteil, Literatur- und Quellenverzeichnis sowie einem Register ergänzt, damit stellt er ein ideales Grundlagenwerk für alle dar, die sich mit dem Umgang mit Kranken im frühneuzeitlichen Europa beschäftigen und ist gleichzeitig auch für eine breitere Leserschaft sehr zu empfehlen. 
